\title{
State Trajectories Analysis for a Class of Tubular Reactor Nonlinear Nonautonomous Models
}

\author{
B. Aylaj, ${ }^{1,2}$ M. E. Achhab, ${ }^{2}$ and M. Laabissi ${ }^{2}$ \\ ${ }^{1}$ Center de Recherche INRIA Futurs-Site de Bordeaux-IMB, 351 Cours de la Libération, \\ 33405 Talent Cedex, Bordeaux, France \\ ${ }^{2}$ Département de Mathématique et Informatique, Faculté des Sciences, Université Chouaib Doukkali, \\ El Jadida, BP 20, Morocco
}

Correspondence should be addressed to B. Aylaj, aylaj@math.u-bordeaux1.fr

Received 4 July 2007; Accepted 3 September 2007

Recommended by Nicholas Dimitrios Alikakos

The existence and uniqueness of global mild solutions are proven for a class of semilinear nonautonomous evolution equations. Moreover, it is shown that the system, under considerations, has a unique steady state. This analysis uses, essentially, the dissipativity, a subtangential condition, and the positivity of the related $C_{0}$-semigroup.

Copyright (C) 2008 B. Aylaj et al. This is an open access article distributed under the Creative Commons Attribution License, which permits unrestricted use, distribution, and reproduction in any medium, provided the original work is properly cited.

\section{Introduction}

Several chemical and biochemical processes are typically described by nonlinear coupled partial differential equations "PDE" and hence by distributed parameter models (see [1] and the references within). The source of nonlinearities is essentially the kinetics of the reactions involved in the process. For numerical simulation as well as for control design problems, many authors approximate those distributed parameter systems by lumped parameter models [15]. However, an important number of questions remained unsolved. In particular, to study the stability of the tubular reactor, the trajectory must exist on the whole real positive time interval $[0,+\infty[$. In our previous works $[6,7]$, we have proven the global state trajectories existence for a class of nonlinear systems arising from convection-dispersion-reaction systems, assuming that the inlet concentrations are independent of time. In this paper, we investigate the question in the case where the involved inlet concentrations are functions of time $t$. The considered class of models correspond to the following chemical reaction:

$$
n A+m B \longrightarrow P,
$$


whose kinetic is given by $r=\left(-k_{1} C^{m} L^{n},-k_{2} C^{m} L^{n}\right)^{T}$, where $C$ and $L$ are the concentrations of the reactants $A$ and $B$, respectively, $k_{1}$ and $k_{2}$ are the kinetic constants and $m, n$ are the order of the reaction to $A$ and $B$, respectively. More precisely, we study the global existence and uniqueness of the trajectories of the models which describe the evolution of two reactant concentrations $C$ and $L$ :

$$
\begin{aligned}
& \frac{\partial C}{\partial t}=-v \frac{\partial C}{\partial \xi}+D_{1} \frac{\partial^{2} C}{\partial \xi^{2}}-k_{1} C^{m} L^{n}, \\
& \frac{\partial L}{\partial t}=-v \frac{\partial L}{\partial \xi}+D_{2} \frac{\partial^{2} L}{\partial \xi^{2}}-k_{2} C^{m} L^{n},
\end{aligned}
$$

for $\xi \in] 0, l[$ and $t>0$, with the following boundary and initial conditions:

$$
\begin{gathered}
D_{1} \frac{\partial C}{\partial \xi}(0, t)-v C(0, t)+v C_{\text {in }}(t)=0=D_{1} \frac{\partial C}{\partial \xi}(l) \quad \forall t>0 \\
D_{2} \frac{\partial L}{\partial \xi}(0, t)-v L(0, t)+v L_{\text {in }}(t)=0=D_{2} \frac{\partial L}{\partial \xi}(l, t) \quad \forall t>0, \\
\left.C(\xi, 0)=C_{0}(\xi), \quad L(\xi, 0)=L_{0}(\xi) \quad \text { for } \xi \in\right] 0, l[.
\end{gathered}
$$

Additionally, the existence and uniqueness of the corresponding equilibrium profile will be proven.

In the above equations, $D_{1}, D_{2}$ are the dispersion coefficients, $v$ is the superficial fluid velocity, $t, \xi$ denote the time and space independent variables, respectively, $l$ is the length of the reactor, $m$ and $n$ are two positive integers, $C_{\text {in }}$ and $L_{\text {in }}$ are the inlet concentration. For further discussion of parameters, we refer to [3].

Comment 1. (i) The nonlinear models considered in this paper have been studied in a qualitative manner by several authors. In the case, $v=0$, [8] established the asymptotic behavior of solutions for the second-order reaction (i.e., $n=m=1$ ). N. Alikakos [9] established global existence and $L^{\infty}$ bounds of positive solutions, when $m=1$ and $1<n<3 / 2$. This latter result has been generalized by [10] for the case $m=1$ and $n>1$.

In practice, the special cases $m=n=1,2,3$ have been used as an industrial pulp bleaching model, where the two reactants are chlorine dioxide $(C)$ and lignin $(L)$. In particular, [3] studied approximate solutions by using several methods (orthogonal collocation, finite elements, and finite difference methods), when $n=m$ and $D_{1}=D_{2}$. The reader can find another model with $D_{1} \neq D_{2}$ in [11], where the numerical analysis has been done for $m=n=1$ and $D_{2}=4 D_{1}, D_{2}=16 D_{1}$ (see also [12]).

Recently, the existence of global solutions for problems such as (1.2)-(1.6) has been extensively studied in $[6,7]$ with constant inlet concentrations.

(ii) For technological limitations and economical considerations, the following saturation conditions are usually fulfilled for all $0 \leq \xi \leq l$ and for all $t \geq 0$ :

$$
\begin{array}{ll}
0 \leq C \leq \bar{C}, & 0 \leq L \leq \bar{L}, \\
C_{\text {in }}(t) \leq \bar{C}, & L_{\text {in }}(t) \leq \bar{L},
\end{array}
$$

where $\bar{C}$ and $\bar{L}$ are positive constants. 
This paper is organized as follows. In Section 2, we will recall briefly some basic results and preliminary facts from semilinear nonautonomous evolution equations which will be used throughout Section 4. In Section 3, the problem (1.2)-(1.6) is converted through some transformations to a homogeneous form where the semigroup theory applies. In Section 4 we establish the main global existence result for system (1.2)-(1.6). We report the existence and uniqueness of equilibrium profiles results in Section 5. Finally, the main conclusions are outlined in Section 6. The background of our approach can be found in [13-16].

\section{Preliminaries}

Let $X$ be a real Banach space with norm $\|\cdot\|, J=[a, b[(a<b \leq+\infty)$, and let $\{\tau(t) ; t \geq 0\}$ be a linear contraction $C_{0}$-semigroup on $X$ generated by $\mathcal{A}$. Let $B$ be a nonlinear continuous operator form $\Omega$ into $X$, where $\Omega$ is a subset of $J \times X$. I and II denote, respectively, the identity operator of $X$ and the function identically equal to 1.

This section is devoted to investigate sufficient conditions for the existence and uniqueness of global mild solutions to the following abstract Cauchy problem:

$$
\begin{gathered}
\dot{x}(t)=\mathcal{A} x(t)+B(t, x(t)), \quad \tau<t<b, \\
x(\tau)=x_{\tau} \in \Omega(\tau),
\end{gathered}
$$

where $\Omega(\tau)$ denote the section of $\Omega$ at $\tau \in J$, given by $\Omega(t)=\{x \in X ;(t, x) \in \Omega\}$. Assume that $\Omega(t) \neq \varnothing$ for all $t \in J$. Moreover, recall that $d(x ; \Phi)=\inf \{\|x-y\|, y \in \Phi\}$, for $x \in X$ and $\Phi$ is a subset of $X$.

The semilinear nonautonomous evolution equations have been treated by a number of authors $[14,15,17-21]$. However, one may find that in most cases $\Omega$ is cylindrical, that is, $\Omega=J \times \Phi[14,22]$. More generally, the cylindrical case of $\Omega$ will not be convenient for the study of evolution system satisfying time-dependent constraints, that is, $x(t) \in \Omega(t)$ on $J$ (see our problem in Section 3). A noncylindrical $\Omega$ case was studied in $[16,19]$.

The following result gives sufficient conditions for the existence and uniqueness of global mild solutions to the semilinear equations of type (2.1). It is a particular version of $\left[16\right.$, Theorem 8.1], when the nonlinear $\boldsymbol{B}(t, \cdot)$ is $l_{\mathcal{B}}$-dissipative [16].

Theorem 2.1 (see [16]). Suppose that the following conditions are fulfilled:

(i) $\Omega$ is closed from the left, that is, if $\left(t_{n}, x_{n}\right) \in \Omega, t_{n} \uparrow t$ in $J$, and $x_{n} \rightarrow x$ in $X$ as $n \rightarrow \infty$, then $(t, x) \in \Omega$;

(ii) for all $(t, x) \in \Omega, \liminf _{h \downarrow 0}(1 / h) d(\tau(h) x+h 乃(t, x), \Omega(t+h))=0$;

(iii) $\boldsymbol{B}$ is continuous on $\Omega$ and there exists $l_{\boldsymbol{B}} \in \mathbb{R}^{+}$such that the operator $\left(\boldsymbol{B}(t, \cdot)-l_{\mathbb{B}} I\right)$ is dissipative on $\Omega(t)$ for all $t \in J$.

If $\Omega$ is a connected subset of $J \times X$ such that for all $t \in J, \Omega(t) \neq \varnothing$, then, for each $\left(\tau, x_{\tau}\right) \in \Omega,(2.1)$ has a unique mild solution on $\mathrm{J}$.

Comment 2. It is shown in [16] that the "subtangential condition" (ii) is a necessary condition for the existence of the mild solutions of (2.1). For more details on the conditions of Theorem 2.1, we refer to [16].

In the particular case when $\Omega(t)$ is $\tau(s)$-invariant, that is, $\tau(s)(\Omega(t)) \subset \Omega(t)$ for all $t, s \geq$ 0 , we have the following lemma. 
Lemma 2.2. Let $\Pi: \Omega \rightarrow X$ be continuous and let $\Omega$ be closed from the left. If $\Omega(t)$ is $\tau(s)$-invariant for all $t, s \geq 0$, then the following subtangential condition

$$
\lim _{h \downarrow 0} \inf \frac{1}{h} d(x+h ß(t, x) ; \Omega(t+h))=0 \quad \forall(t, x) \in \Omega
$$

implies the condition

$$
\liminf _{h \downarrow 0} \frac{1}{h} d(\tau(h) x+h ß(t, x), \Omega(t+h))=0, \quad \forall(t, x) \in \Omega
$$

Proof. Let $(t, x) \in \Omega$, given $\epsilon>0$, from condition (2.2) it follows, by [23, Lemma 3] (see also [24, Lemma 1]), that there is $h \in(0, \epsilon]$ and $y \in \Omega(t+h)$ such that $\|y-x-h \beta(t, x)\| \leq h \epsilon$. Let now $u=y-x-h B(t, x)$ and $v=(1 / h) u$. We get $\|v\| \leq \epsilon$ such that $y=x+h(B(t, x)+v) \in \Omega(t+h)$. By the invariance properties of $\Omega(t)$, we have $\tau(h) y \in \Omega(t+h)$. Consequently,

$$
\begin{aligned}
& d(\tau(h) x+h 乃(t, x) ; \Omega(t+h)) \leq\|\tau(h) x+h ß(t, x)-\tau(h) y\|, \\
& \leq\|h ß(t, x)-h \tau(h) ß(t, x)-h \tau(h) v\|, \\
& \leq h\|\tau(h) B(t, x)-\beta(t, x)\|+h\|\tau(h) v\|, \\
& \leq h\|\tau(h) ß(t, x)-\beta(t, x)\|+h \epsilon \text {. }
\end{aligned}
$$

By using the continuity of $C_{0}$-semigroup $(\tau(t))_{t \geq 0}$, the desired result (2.3) is obtained.

Theorem 2.1 with Lemma 2.2 obviously imply the following.

Corollary 2.3. Suppose that the following conditions are fulfilled:

(i) $\Omega$ is closed from the left, that is, if $\left(t_{n}, x_{n}\right) \in \Omega, t_{n} \uparrow t$ in $J$, and $x_{n} \rightarrow x$ in $X$ as $n \rightarrow \infty$, then $(t, x) \in \Omega$;

(ii) $\Omega(t)$ is $\tau(s)$-invariant, for all $t, s \geq 0$;

(iii) for all $(t, x) \in \Omega, \liminf _{h \downarrow 0}(1 / h) d(x+h \beta(t, x), \Omega(t+h))=0$;

(iv) $\boldsymbol{B}$ is continuous on $\Omega$ and there exists $l_{B} \in \mathbb{R}^{+}$such that the operator $\left(B(t, \cdot)-l_{B} I\right)$ is dissipative on $\Omega(t)$, for all $t \in J$.

If $\Omega$ is a connected subset of $J \times X$ such that for all $t \in J, \Omega(t) \neq \varnothing$, then, for each $\left(\tau, x_{\tau}\right) \in \Omega,(2.1)$ has a unique mild solution on $J$.

\section{Abstract semigroup formulation}

Throughout the sequel, we assume $H=L^{2}[0,1] \oplus L^{2}[0,1]$, the Hilbert space with the usual inner product

$$
\left\langle\left(x_{1}, x_{2}\right),\left(y_{1}, y_{2}\right)\right\rangle=\left\langle x_{1}, y_{1}\right\rangle_{L^{2}}+\left\langle x_{2}, y_{2}\right\rangle_{L^{2}}
$$


and the induced norm

$$
\left\|\left(x_{1}, x_{2}\right)\right\|=\left(\left\|x_{1}\right\|_{L^{2}}^{2}+\left\|x_{2}\right\|_{L^{2}}^{2}\right)^{1 / 2}
$$

for all $\left(x_{1}, x_{2}\right)$ and $\left(y_{1}, y_{2}\right)$ in $H$.

Clearly, the Hilbert space $H$ is a real Banach lattice, where for all given $x=\left(x_{1}, x_{2}\right) \in$ $H, y=\left(y_{1}, y_{2}\right) \in H$,

$$
x \leq y \quad \text { iff } x_{1}(z) \leq y_{1}(z), \quad x_{2}(z) \leq y_{2}(z) \quad \text { for a.e. } z \in[0,1] .
$$

Recall that for every pair $x, y \in H$, the set

$$
[x, y]=\left\{w \in H: x_{1} \leq w_{1} \leq y_{1}, x_{2} \leq w_{2} \leq y_{2}\right\}=\left[x_{1}, y_{1}\right] \times\left[x_{2}, y_{2}\right]
$$

is called the order interval between $x$ and $y$. Clearly, $[x, y]$ is nonempty if $x \leq y$ (for more details, see, e.g., [25]). A bounded linear operator $\tau$ on $H$ is said to be positive if $0 \leq \tau x$ for all $0 \leq x$. Similarly, a family of bounded linear operators $(\tau(t))_{t \geq 0}$ of $H$ is said to be a positive $C_{0}$-semigroup on $H$ if $\tau(t)$ is a $C_{0}$-semigroup on $H$ and $\tau(t)$ is a positive operator for all $t \geq 0$.

In the following, we will assume that $C_{\text {in }}(t)$ and $L_{\text {in }}(t)$ are positive $\mathcal{C}^{1}([0, \infty[)$-functions. Let us consider the following state transformation:

$$
z=\frac{\xi}{l}, \quad x_{1}=C-C_{\mathrm{in}}, \quad x_{2}=L-L_{\mathrm{in}}, \quad x_{01}=C_{0}-C_{\mathrm{in}}, \quad x_{02}=L_{0}-L_{\mathrm{in}} .
$$

Then, we obtain the new equivalent system for all $z \in] 0,1[$ and $t>0$ :

$$
\begin{aligned}
& \frac{\partial x_{1}}{\partial t}=-v \frac{\partial x_{1}}{\partial z}+d_{1} \frac{\partial^{2} x_{1}}{\partial z^{2}}-k_{1}\left(x_{1}+C_{\text {in }}(t)\right)^{m}\left(x_{2}+L_{\text {in }}(t)\right)^{n}-\dot{C_{\text {in }}}(t), \\
& \frac{\partial x_{2}}{\partial t}=-v \frac{\partial x_{2}}{\partial z}+d_{2} \frac{\partial^{2} x_{2}}{\partial z^{2}}-k_{2}\left(x_{1}+C_{\text {in }}(t)\right)^{m}\left(x_{2}+L_{\text {in }}(t)\right)^{n}-\dot{L_{\text {in }}}(t),
\end{aligned}
$$

with

$$
\begin{gathered}
d_{i} \frac{\partial x_{i}}{\partial z}(0, t)-v x_{i}(0, t)=0=d_{i} \frac{\partial x_{i}}{\partial z}(1, t) \quad \forall t>0 i=1 ; 2 \\
\left.x_{i}(z, 0)=x_{0 i}(z) \quad \text { for } z \in\right] 0,1[, i=1 ; 2
\end{gathered}
$$

where

$$
d_{1}=\frac{D_{1}}{l^{2}}, \quad d_{2}=\frac{D_{2}}{l^{2}}, \quad v=\frac{v}{l}
$$

This PDEs describing the reactor dynamics may be formally written in the abstract form as

$$
\begin{gathered}
\dot{x}(t)=A x(t)+B(t, x(t)), \\
x(0)=x_{0} \in \Omega(0),
\end{gathered}
$$


where $\Omega(t)$ denote the section of $\Omega$ at $t \in \mathbb{R}^{+}$, which is given in view of (1.7) by

$$
\begin{aligned}
\Omega=\{ & \left(t,\left(x_{1}, x_{2}\right)\right)^{T} \in \mathbb{R}^{+} \times H:-C_{\text {in }}(t) \leq x_{1}(z) \leq \bar{C}-C_{\text {in }}(t), \\
& \left.-L_{\text {in }}(t) \leq x_{2}(z) \leq \bar{L}-L_{\text {in }}(t) \text { a.e. } z \in[0,1]\right\} .
\end{aligned}
$$

The linear operator $A$ is defined by

$$
\begin{aligned}
& D(A)=\left\{x=\left(x_{1}, x_{2}\right)^{T} \in H: x, \frac{d x}{d z} \in H\right. \text { are absolutely continuous, } \\
& \left.\frac{d^{2} x}{d z^{2}} \in H, d_{i} \frac{d x_{i}}{d z}(0)-v x_{i}(0)=0=d_{i} \frac{d x_{i}}{d z}(1) ; i=1 ; 2\right\}, \\
& A x=\left(\begin{array}{cc}
d_{1} \frac{d^{2} x_{1}}{d z^{2}}-v \frac{d x_{1}}{d z} & 0 \\
0 & d_{2} \frac{d^{2} x_{2}}{d z^{2}}-v \frac{d x_{2}}{d z}
\end{array}\right)=\left(\begin{array}{cc}
A_{1} x_{1} & 0 \\
0 & A_{2} x_{2}
\end{array}\right) .
\end{aligned}
$$

The nonlinear operator $B$ is defined on $\Omega$ by

$$
\begin{aligned}
B(t, x)=( & -k_{1}\left(x_{1}+C_{\mathrm{in}}(t) \mathbb{I}\right)^{m}\left(x_{2}+L_{\mathrm{in}}(t) \mathbb{I}\right)^{n}-\dot{C_{\mathrm{in}}}(t) \mathbb{I}, \\
& \left.-k_{2}\left(x_{1}+C_{\mathrm{in}}(t) \mathbb{I}\right)^{m}\left(x_{2}+L_{\mathrm{in}}(t) \mathbb{I}\right)^{n}-\dot{L_{\mathrm{in}}}(t) \mathbb{I}\right)^{T} .
\end{aligned}
$$

It is shown in [7] that the linear operator $A$ given by (3.14) is the infinitesimal generator of contraction semigroup on $H$

$$
T(t)=\left(\begin{array}{cc}
T_{1}(t) & 0 \\
0 & T_{2}(t)
\end{array}\right),
$$

where $T_{1}(t)$ and $T_{2}(t)$ are the $C_{0}$-semigroups generated, respectively, by $A_{1}$ and $A_{2}$.

\section{Global existence}

This section is concerned with the existence and the uniqueness of mild solution for our problem given by (3.6)-(3.9) In order to be able to apply Corollary 2.3, we need the following lemmas.

Lemma 4.1. For each $(t, x) \in \Omega$,

$$
\lim _{h \downarrow 0} \frac{1}{h} d(x+h B(t, x) ; \Omega(t+h))=0 .
$$

Proof. Let $(t, x) \in \Omega$. Observe that $\Omega(t)$ is given by $\Omega(t)=\Omega_{1}(t) \times \Omega_{2}(t)$, where

$$
\begin{aligned}
& \Omega_{1}(t)=\left[-C_{\text {in }}(t) \mathbb{I},\left(\bar{C}-C_{\text {in }}(t)\right) \mathbb{I}\right], \\
& \Omega_{2}(t)=\left[-L_{\text {in }}(t) \mathbb{I},\left(\bar{L}-L_{\text {in }}(t)\right) \mathbb{I}\right] .
\end{aligned}
$$


Denote

$$
X_{1}(t)=x_{1}+C_{\text {in }}(t) \mathbb{I}, \quad X_{2}(t)=x_{2}+L_{\text {in }}(t) \mathbb{I},
$$

we have, for $x \in \Omega(t)$,

$$
X(t)=\left(X_{1}(t), X_{2}(t)\right)^{T} \in[0, \bar{C} \mathbb{I}] \times[0, \bar{L} \mathbb{I}]
$$

Let $h_{0}>0$ be sufficiently small such that $h_{0} k_{1} \bar{C}^{m-1} \bar{L}^{n} \leq 1$.

Let, now, $h \in\left(0, h_{0}\right)$, then

$$
X_{1}(t)\left(\mathbb{I}-h k_{1} X_{1}^{m-1}(t) X_{2}^{n}(t)\right) \in[0, \bar{C} \mathbb{I}] .
$$

Hence

$$
f_{1}(t, X(t))=X_{1}(t)\left(\mathbb{I}-h k_{1} X_{1}^{m-1}(t) X_{2}^{n}(t)\right)-C_{\text {in }}(t+h) \mathbb{I} \in \Omega_{1}(t+h) .
$$

By using the regularity of the inlet function $C_{\text {in }}$, we get

$$
\begin{aligned}
d\left(x_{1}+h B_{1}(t, x), \Omega_{1}(t+h)\right) & \leq d\left(X_{1}(t)-h k_{1} X_{1}^{m}(t) X_{2}^{n}(t)-C_{\text {in }}(t+h) \mathbb{I}, \Omega_{1}(t+h)\right)+h \epsilon(h) \\
& \leq d\left(f_{1}(t, X(t)), \Omega_{1}(t+h)\right)+h \epsilon(h)=h \epsilon(h),
\end{aligned}
$$

where $\epsilon(h) \rightarrow 0$ as $h \rightarrow 0$. Whence

$$
\lim _{h \downarrow 0} \frac{1}{h} d\left(x_{1}+h B_{1}(t, x) ; \Omega_{1}(t+h)\right)=0 .
$$

By similar considerations as above, taking into account the regularity of the function $L_{\mathrm{in}}$, we also get

$$
\lim _{h \downarrow 0} \frac{1}{h} d\left(x_{2}+h B_{2}(t, x) ; \Omega_{2}(t+h)\right)=0 .
$$

Observe, now, that

$$
d(x+B(t, x), \Omega(t+h)) \leq d\left(x_{1}+B_{1}(t, x), \Omega_{1}(t+h)\right)+d\left(x_{2}+B_{2}(t, x), \Omega_{2}(t+h)\right),
$$

combining the latter with (4.8)-(4.9) we get the desired result (4.1).

The following lemma is useful to establish the dissipativity property.

Lemma 4.2. There exists $l_{B} \in \mathbb{R}^{+}$such that the operator $\left(B(t, \cdot)-l_{B} I\right)$ is dissipative on $\Omega(t)$ for each $t \geq 0$. 
Proof. Let $t \geq 0$ and let $x, y$ be in $\Omega(t)$. Denote

$$
g_{i}(t, x)=-k_{i}\left(x_{1}+C_{\text {in }}(t) \mathbb{I}\right)^{m}\left(x_{2}+L_{\text {in }}(t) \mathbb{I}\right)^{n} \quad \text { for } i=1,2,
$$

and let also

$$
X_{1}(t)=x_{1}+C_{\text {in }}(t) \mathbb{I} ; \quad X_{2}(t)=x_{2}+L_{\text {in }}(t) \mathbb{I} ; \quad Y_{1}(t)=y_{1}+C_{\text {in }}(t) \mathbb{I}, \quad Y_{2}(t)=y_{2}+L_{\text {in }}(t) \mathbb{I} .
$$

Observe that, for each $x, y \in \Omega(t),\left(X_{i}(t), Y_{i}(t)\right)^{T} \in[0, \bar{C} \mathbb{I}] \times[0, \bar{L} \mathbb{I}]$ for $i=1,2$. Hence, by applying the mean value theorem, for $i=1,2$, we get

$$
\begin{aligned}
\left\|g_{i}(t, x)-g_{i}(t, y)\right\|_{L^{2}} & \leq k_{i}\left(\bar{C}^{2 m}\left\|X_{2}^{n}(t)-Y_{2}^{n}(t)\right\|_{L^{2}}^{2}+\bar{L}^{2 n}\left\|X_{1}^{m}(t)-Y_{1}^{m}(t)\right\|_{L^{2}}^{2}\right)^{1 / 2} \\
& \leq k_{i}\left(n^{2} \bar{C}^{2 m} \bar{L}^{2 n-2}\left\|x_{2}-y_{2}\right\|_{L^{2}}^{2}+m^{2} \bar{L}^{2 n} \bar{C}^{2 m-2}\left\|x_{1}-y_{1}\right\|_{L^{2}}^{2}\right)^{1 / 2} \\
& \leq k_{i} \bar{C}^{m-1} \bar{L}^{n-1} \max (n \bar{C} ; m \bar{L})\|x-y\| .
\end{aligned}
$$

Finally,

$$
\begin{aligned}
\|B(t, x)-B(t, y)\| & =\left(\left\|g_{1}(t, x)-g_{1}(t, y)\right\|_{L^{2}}^{2}+\left\|g_{2}(t, x)-g_{2}(t, y)\right\|_{L^{2}}^{2}\right)^{1 / 2} \\
& \leq \max \left(k_{1}, k_{2}\right) \bar{C}^{m-1} \bar{L}^{n-1} \max (n \bar{C} ; m \bar{L})\|x-y\| .
\end{aligned}
$$

Consequently, $B(t, \cdot)$ is an $l_{B}$-dissipative operator on $\Omega(t)$ [14, page 245], where

$$
l_{B}=\max \left(k_{1}, k_{2}\right) \bar{C}^{m-1} \bar{L}^{n-1} \max (n \bar{C} ; m \bar{L}) .
$$

Finally, we state the invariance properties of the state trajectories of the model given by (3.6)-(3.9).

Proposition 4.3. One has that

$$
\Omega(t) \text { is } \tau(s) \text {-invariant } \forall t, s \geq 0 .
$$

Proof. Let $t, s \geq 0$ and $(x, y)^{T} \in \Omega(t)$. We have

$$
\left(-C_{\text {in }}(t) \mathbb{I},-L_{\text {in }}(t) \mathbb{I}\right)^{T} \leq(x, y)^{T} \leq\left(\left(\bar{C}-C_{\text {in }}(t)\right) \mathbb{I},\left(\bar{L}-L_{\text {in }}(t)\right) \mathbb{I}\right)^{T} .
$$

Hence, by using the positivity of $(T(t))_{t \geq 0}$ [26], we have

$$
\begin{aligned}
\left(-C_{\text {in }}(t) T_{1}(s) \mathbb{I},-L_{\text {in }}(t) T_{2}(s) \mathbb{I}\right)^{T} & \leq T(s)(x, y)^{T} \\
& \leq\left(\left(\bar{C}-C_{\text {in }}(t)\right) T_{1}(s) \mathbb{I},\left(\bar{C}-C_{\text {in }}(t)\right) T_{2}(s) \mathbb{I}\right)^{T} .
\end{aligned}
$$

Since, $T_{i}(t) \mathbb{I} \leq \mathbb{I}$ for $i=1 ; 2$ (see [26]) and by using the inequalities (1.8) (i.e., $\bar{C} \geq C_{\text {in }}$ and $\left.\bar{L} \geq L_{\text {in }}\right)$, the invariance of $\Omega(t)$ holds for all $t \geq 0$. Thus, $\left(T_{1}(s) x, T_{2}(s) y\right)^{T} \in \Omega(t)$ for all $t, s \geq 0$. 
Now, we are in a position to state and prove our global existence result for problem (3.6)-(3.9).

Theorem 4.4. Let $C_{\text {in }}(t)$ and $L_{\text {in }}(t)$ be positive $C^{1}\left(\left[0,+\infty[)\right.\right.$-functions. Then, for every $x_{0} \in \Omega(0)$, the problem (3.6)-(3.9) has a unique global mild solution.

Proof. Since $B$ is continuous function in $\Omega$, by Corollary 2.3, it is sufficient to prove the condition (i) in Corollary 2.3 and to check that the subset $\Omega$ is connected

(a) Let us first show that $\Omega$ is closed from the left.

Let $t_{n} \nearrow t$ and $x_{n} \in \Omega\left(t_{n}\right)$ with $x_{n} \rightarrow x \in H$, then there exists a subsequence of $\left(x_{n}\right)$ which is also denoted by $\left(x_{n}\right)$ such that $x_{n}(z) \rightarrow x(z)$, that is, on $[0,1]$ which implies, by continuity of $C_{\text {in }}$ and $L_{\text {in }}$, that $x(z) \in\left[-C_{\text {in }}(t), \bar{C}-C_{\text {in }}(t)\right] \times\left[-L_{\text {in }}(t), \bar{L}-L_{\text {in }}(t)\right]$, that is, on $[0,1]$, hence $x \in \Omega(t)$ for each $t \geq 0$.

(b) Let us, now, check that $\Omega$ is connected in $[0,+\infty[\times H$ :

Let $K=[0, \bar{C} \mathbb{I}] \times[0, \bar{L} \mathbb{I}]$ and define $G:[0,+\infty[\times K \rightarrow \Omega$ such that for all $(t, x) \in$ $\left[0,+\infty\left[\times K, G(t, x)=\left(t, x_{1}-C_{\text {in }}(t) \mathbb{I}, x_{2}-L_{\text {in }}(t) \mathbb{I}\right)^{T}\right.\right.$. Since $C_{\text {in }}$ and $L_{\text {in }}$ are continuous functions in $[0,+\infty[$, it follows that $G$ in $[0,+1[\times K$ is also a continuous function. Observe that $G$ is surjective; since $[0, \bar{C} \mathbb{I}] \times[0, \bar{L} \mathbb{I}]$ is connected in $H$, we get that $\Omega=G([0,+\infty[\times K)$ is also connected in $[0,+\infty[\times H$.

Thus the proof of the theorem is complete.

The next section deals with the existence and uniqueness results of equilibrium profile solutions for a nonlinear model given by (3.6)-(3.9).

\section{Equilibrium profiles}

In the steady-state solution analysis, the inlet functions $C_{\text {in }}$ and $L_{\text {in }}$ are independent of time $t$, which implies that the domain $\Omega(t)$ is also independent of $t$. If we denote by $\overline{C_{\text {in }}}$ and $\overline{L_{\text {in }}}$ the values of $C_{\text {in }}$ and $L_{\text {in }}$ which correspond to the steady-state solutions, the corresponding steady-state system to the models (3.6)-(3.9) is given by the following equations:

$$
\begin{aligned}
& -v \frac{d x_{1}}{d z}=d_{1} \frac{d^{2} x_{1}}{d z^{2}}-k_{1}\left(x_{1}+\overline{C_{\text {in }}}\right)^{m}\left(x_{2}+\overline{L_{\text {in }}}\right)^{n}=0, \\
& -v \frac{d x_{2}}{d z}=d_{2} \frac{d^{2} x_{2}}{d z^{2}}-k_{2}\left(x_{1}+\overline{C_{\text {in }}}\right)^{m}\left(x_{2}+\overline{L_{\text {in }}}\right)^{n}=0,
\end{aligned}
$$

with

$$
\begin{gathered}
d_{i} \frac{d x_{i}}{d z}(0)-v x_{i}(0)=0=d_{i} \frac{d x_{i}}{d z}(1), \quad i=1 ; 2, \\
\Omega(t)=\Delta=\left\{\left(x_{1}, x_{2}\right)^{T} \in H:-\overline{C_{\mathrm{in}}} \leq x_{1}(z) \leq \bar{C}-\overline{C_{\mathrm{in}}}\right. \\
\left.-\overline{L_{\mathrm{in}}} \leq x_{2}(z) \leq \bar{L}-\overline{L_{\mathrm{in}}} \text { for almost all } z \in[0,1]\right\} .
\end{gathered}
$$

The following existence result can be proven as in the case where $C_{\mathrm{in}}$ and $L_{\mathrm{in}}$ are independent of time. 
Theorem 5.1 (see $[7,27])$. The tubular reactor modelled by the nonlinear coupled partial differential equations given by (3.6)-(3.9) has at least one equilibrium profile in $\Delta$.

The sequel of this paper will deal with the uniqueness analysis of steady states in the important case where $d_{1}=d_{2}=d$.

First, since $d_{1}=d_{2}=d$, we denote $\mathscr{A}=d\left(d^{2} / d z^{2}\right)-v(d / d z)=A_{i}$ with $D(\mathcal{A})=D\left(A_{i}\right)$ for $i=1 ; 2$.

Now, we derive a positivity lemma, which will play a fundamental role in the proof of the uniqueness result of steady states.

Lemma 5.2. Let $b(\cdot)$ be a bounded nonnegative function defined in $[0,1]$. If $u \in L^{2}([0,1])$ satisfies the equations

$$
\mathcal{A} u=b u \text { in }] 0,1[, u \in D(\mathscr{A}) \text {, }
$$

then $u=0$ in $[0,1]$

Proof. Let $u$ be the solution of problem (5.5), then

$$
\langle\mathcal{A} u, u\rangle_{L^{2}}=\langle b u, u\rangle_{L^{2}}
$$

We have,

$$
\begin{aligned}
\langle\mathcal{A} u, u\rangle_{L^{2}} & =\int_{0}^{1}\left[d \frac{d^{2} u}{d z^{2}}(z)-v \frac{d u}{d z}(z)\right] u(z) d z \\
& =-\int_{0}^{1} d\left[\frac{d u}{d z}(z)\right]^{2} d z+d\left[\frac{d u}{d z}(1) u(1)-\frac{d u}{d z}(0) u(0)\right]-\frac{1}{2} v\left[u^{2}(1)-u^{2}(0)\right] \\
& =-d\left\|\frac{d u}{d z}\right\|_{L^{2}}^{2}-\frac{1}{2} v u^{2}(1)-\frac{1}{2} v u^{2}(0) \\
& \leq 0
\end{aligned}
$$

Since $b(z)$ is nonnegative function in $[0,1]$, then by (5.8) and taking into account (5.6)

$$
\langle b u, u\rangle_{L^{2}}=\int_{0}^{1} b(z) u^{2}(z) d z=0 .
$$

Which implies, in view of (5.6)-(5.7), that

$$
\langle\mathcal{A} u, u\rangle_{L^{2}}=0=d\left\|\frac{d u}{d z}\right\|_{L^{2}}^{2}+\frac{1}{2} v u^{2}(1)+\frac{1}{2} v u^{2}(0) .
$$

Then, we get

$$
\frac{d u}{d z}(z)=0 \quad \text { a.e. } z \in[0,1]
$$

Clearly, by using the Sobolev imbedding theorem, $D(\mathscr{A}) \subset \mathcal{C}([0,1])$. Therefore, $u=0$ since $u \in D(\mathcal{A})$. 
Theorem 5.3. For $d_{1}=d_{2}=d$, the steady-state problem given by (5.1)-(5.3) has a unique solution in $\Delta$.

Proof. Let $x=\left(x_{1}, x_{2}\right)^{T}$ and $y=\left(y_{1}, y_{2}\right)^{T}$ be solutions to (5.1)-(5.3) on [0,1]. To obtain the desired result, we will be showing that $x=y$. Let

$$
\begin{gathered}
g\left(x_{1}, x_{2}\right)=-\left(x_{1}+\overline{C_{\text {in }}} \mathbb{I}\right)^{m}\left(x_{2}+\overline{L_{\text {in }}} \mathbb{I}\right)^{n}, \\
w_{1}=y_{1}-x_{1} \in D(\mathcal{A}), \quad w_{2}=x_{2}-y_{2} \in D(\mathcal{A}) .
\end{gathered}
$$

Then

$$
\begin{aligned}
-A w_{1}= & k_{1}\left(g\left(y_{1}, y_{2}\right)-g\left(x_{1}, x_{2}\right)\right) \\
= & k_{1}\left(y_{1}+\overline{C_{\text {in }}}\right)^{m}\left[\left(x_{2}+\overline{L_{\text {in }}}\right)^{n}-\left(y_{2}+\overline{L_{\text {in }}}\right)^{n}\right] \\
& +k_{1}\left(x_{2}+\overline{L_{\text {in }}}\right)^{n}\left[\left(x_{1}+\overline{C_{\text {in }}}\right)^{m}-\left(y_{1}+\overline{C_{\text {in }}}\right)^{m}\right]
\end{aligned}
$$

Hence, by applying the mean value theorem, we get

$$
-A w_{1}=k_{1} n\left(y_{1}+\overline{C_{\text {in }}}\right)^{m} \xi_{2}^{n-1} w_{2}-m k_{1}\left(x_{2}+\overline{L_{\text {in }}}\right)^{n} \xi_{1}^{m-1} w_{1},
$$

where $\left(\xi_{1}, \xi_{2}\right)$ are some intermediate values between $(0,0)$ and $(\bar{C}, \bar{L})$.

By similar considerations as above, we also get

$$
\begin{aligned}
-A w_{2} & =-k_{2}\left(g\left(y_{1}, y_{2}\right)-g\left(x_{1}, x_{2}\right)\right) \\
& =-k_{2} n\left(y_{1}+\overline{C_{\text {in }}}\right)^{m} \xi_{2}^{n-1} w_{2}+m k_{2}\left(x_{2}+\overline{L_{\text {in }}}\right)^{n} \xi_{1}^{m-1} w_{1},
\end{aligned}
$$

for the same $\xi_{1}$ and $\xi_{2}$.

Now, we have the following system:

$$
\begin{aligned}
& -\mathcal{A} w_{1}=-a_{1} w_{1}+b_{1} w_{2}, \\
& -\mathcal{A} w_{2}=a_{2} w_{1}-b_{2} w_{2},
\end{aligned}
$$

where, for $i=1 ; 2$,

$$
\begin{aligned}
& a_{i}(z)=m k_{i}\left(x_{2}(z)+\overline{L_{\mathrm{in}}}\right)^{n} \xi_{1}^{m-1}(z), \\
& b_{i}(z)=n k_{i}\left(y_{1}(z)+\overline{C_{\text {in }}}\right)^{m} \xi_{2}^{n-1}(z) .
\end{aligned}
$$

Multiplying (5.16) by $k_{2}$ and (5.17) by $k_{1}$, we get by addition of both equations that

$$
\mathcal{A} w=0, w \in D(\mathcal{A}),
$$

where $w=k_{2} w_{1}+k_{1} w_{2}$. By Lemma 5.2, this system has a unique solution $w=0$ in $[0,1]$. Now, let

$$
-\mathcal{A} w_{2}=a_{2} w_{1}-b_{2} w_{2}
$$

and substituting the expression

$$
w_{1}=-k_{2}^{-1} k_{1} w_{2}
$$

yields

$$
A w_{2}=c w_{2}
$$


where $c(z)=a_{1}(z)+b_{2}(z)$. Observe that, for $i=1 ; 2$,

$$
0 \leq a_{i}(z) \leq m k_{i} \bar{L}^{n} \bar{C}^{m-1}, \quad 0 \leq b_{i}(z) \leq n k_{i} \bar{C}^{m} \bar{L}^{n-1} .
$$

Let $\lambda=\max (m \bar{L}, n \bar{C}) \max \left(k_{1}, k_{2}\right) \bar{C}^{m-1} \bar{L}^{n-1}$, then we have $0 \leq c(z) \leq 2 \lambda$. By Lemma 5.2 we get $w_{2}=0$. Thus it follows, by (5.21), that $w_{1}=0$, which ensures the desired result, that is, $x=y$.

\section{Conclusion}

In this paper, we have studied the existence and uniqueness of the global mild solution for a class of tubular reactor nonlinear nonautonomous models. It has also been proven that the trajectories are satisfying time-dependent constraints, that is, $x(t) \in \Omega(t)$. Moreover, the set of physically meaningful admissible states $\Omega(t)$ is invariant under the dynamics of the reactions. In addition, the existence and uniqueness results of equilibrium profiles are reported.

An important open question is the stability analysis of equilibrium profile for system (1.2)-(1.6). This question is under investigation.

\section{Acknowledgments}

This paper presents research results of the Moroccan "Programme Thématique d'Appui à la Recherche Scientifique" PROTARS III, initiated by the Moroccan "Centre National de la Recherche Scientifique et Technique" (CNRST). The scientific responsibility rests with its authors. The work has been partially carried out within the framework of a collaboration agreement between CESAME (Université Catholique de Louvain, Belgium) and LINMA of the Faculty of sciences (Univesité Chouaib Doukkali, Morocco), funded by the Belgian Secretary of the State for Development Cooperation and by the CIUF (Conseil Interuniversitaire de la Communauté Française, Belgium). The work of B. Aylaj is supported by a research grant from the Agence Universitaire de la Francophonie.

\section{References}

[1] D. Dochain, Contribution to the analysis and control of distributed parameter systems with application to (bio)chemical processes and robotics, Ph.D. thesis, Universite Catholique de Louvain, Louvain-la-Neuve, Belgium, 1994.

[2] W. H. Ray, Advanced Process Control, Butterworths Series in Chemical Engineering, McGraw-Hill, Boston, Mass, USA, 1981.

[3] S. Renou, Commande Non-Linéaire d'un Systeme Décrit par des Equations Paraboliques: Application au Procédé de Blanchiment, Ph.D. thesis, Génie Chimique, Ecole Polytechnique de Montreal, Montreal, QC, Canada, 2000.

[4] S. Renou, M. Perrier, D. Dochain, and S. Gendron, "Solution of the convection-dispersion-reaction equation by a sequencing method," Computers $\mathcal{E}$ Chemical Engineering, vol. 27, no. 5, pp. 615-629, 2003.

[5] J. J. Winkin, D. Dochain, and P. Ligarius, "Dynamical analysis of distributed parameter tubular reactors," Automatica, vol. 36, no. 3, pp. 349-361, 2000.

[6] M. E. Achhab, B. Aylaj, and M. Laabissi, "Global existence of state trajectoties for a class of tubular reactor nonlinear models," in Proceedings CD-ROM of the 16th International Symposium on the Mathematical Theory of Networks and Systems (MTNS '04), Leuven, Belgium, July 2004. 
[7] B. Aylaj, M. E. Achhab, and M. Laabissi, "Asymptotic behaviour of state trajectories for a class of tubular reactor nonlinear models," IMA Journal of Mathematical Control and Information, vol. 24, no. 2, pp. 163-175, 2007.

[8] R. H. Martin Jr., "Mathematical models in gas-liquid reactions," Nonlinear Analysis. Theory, Methods $\mathcal{E}$ Applications, vol. 4, no. 3, pp. 509-527, 1980.

[9] N. D. Alikakos, "L $L^{p}$ Bounds of solutions of reaction-diffusion equations," Communications in Partial Differential Equations, vol. 4, no. 8, pp. 827-868, 1979.

[10] K. Masuda, "On the global existence and asymptotic behavior of solutions of reaction-diffusion equations," Hokkaido Mathematical Journal, vol. 12, no. 3, pp. 360-370, 1983.

[11] E. P. Van Elk, Gas-liquid reactions: influence of liquid bulk and mass transfer on process performance, Ph.D. thesis, University of Twente, Enschede, The Netherlands, 2001.

[12] P. V. Danckwerts, Gas-Liquid Reactions, McGraw-Hill, New York, NY, USA, 1970.

[13] R. F. Curtain and H. Zwart, An Introduction to Infinite-Dimensional Linear Systems Theory, vol. 21 of Texts in Applied Mathematics, Springer, New York, NY, USA, 1995.

[14] R. H. Martin Jr., Nonlinear Operators and Differential Equations in Banach Spaces, Pure and Applied Mathematics, John Wiley \& Sons, New York, NY, USA, 1976.

[15] A. Pazy, Semigroups of Linear Operators and Applications to Partial Differential Equations, vol. 44 of Applied Mathematical Sciences, Springer, New York, NY, USA, 1983.

[16] T. Iwamiya, "Global existence of mild solutions to semilinear differential equations in Banach spaces," Hiroshima Mathematical Journal, vol. 16, no. 3, pp. 499-530, 1986.

[17] B. Aulbach and N. Van Minh, "Nonlinear semigroups and the existence and stability of solutions of semilinear nonautonomous evolution equations," Abstract and Applied Analysis, vol. 1, no. 4, pp. 351380, 1996.

[18] V. Barbu, Nonlinear semigroups and differential equations in Banach spaces, Editura Academiei Republicii Socialiste România, Bucharest, 1976.

[19] D. Bothe, "Flow invariance for perturbed nonlinear evolution equations," Abstract and Applied Analysis, vol. 1, no. 4, pp. 417-433, 1996.

[20] Z.-H. Luo, B.-Z. Guo, and O. Morgul, Stability and Stabilization of Infinite Dimensional Systems with Applications, Communications and Control Engineering Series, Springer, London, UK, 1999.

[21] C. V. Pao, Nonlinear Parabolic and Elliptic Equations, Plenum Press, New York, NY, USA, 1992.

[22] N. Pavel, "Invariant sets for a class of semi-linear equations of evolution," Nonlinear Analysis. Theory, Methods E Applications, vol. 1, no. 2, pp. 187-196, 1977.

[23] T. Iwamiya, "Global existence of solutions to nonautonomous differential equations in Banach spaces," Hiroshima Mathematical Journal, vol. 13, no. 1, pp. 65-81, 1983.

[24] N. Kenmochi and T. Takahashi, "Nonautonomous differential equations in Banach spaces," Nonlinear Analysis. Theory, Methods \& Applications, vol. 4, no. 6, pp. 1109-1121, 1980.

[25] W. Arendt, A. Grabosch, G. Greiner, et al., One-Parameter Semigroups of Positive Operators, vol. 1184 of Lecture Notes in Mathematics, Springer, Berlin, Germany, 1986.

[26] M. Laabissi, M. E. Achhab, J. J. Winkin, and D. Dochain, "Multiple equilibrium profiles for nonisothermal tubular reactor nonlinear models," Dynamics of Continuous, Discrete E Impulsive Systems. Series B. Applications \& Algorithms, vol. 11, no. 3, pp. 339-352, 2004.

[27] M. E. Achhab, B. Aylaj, and M. Laabissi, "Equilibrium profiles for a class of tubular reactor nonlinear models," in Proceedings of the 13th Mediteranean Conference on Control and Automation (MED '05), Limassol, Cyprus, June 2005. 


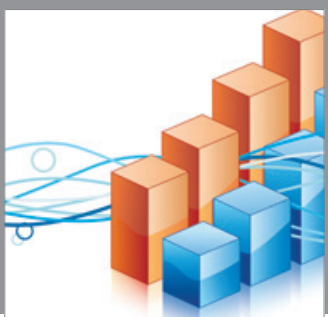

Advances in

Operations Research

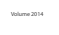

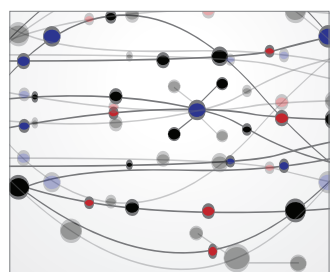

\section{The Scientific} World Journal
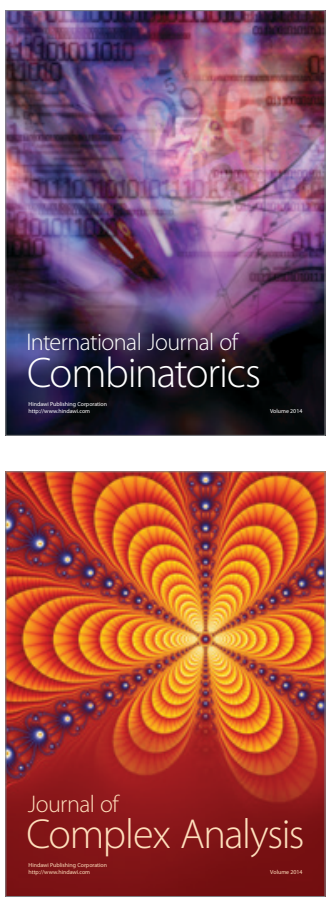

International Journal of

Mathematics and

Mathematical

Sciences
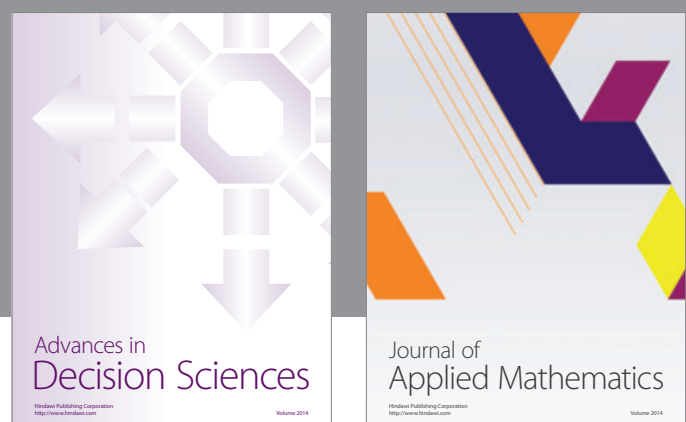

Journal of

Applied Mathematics
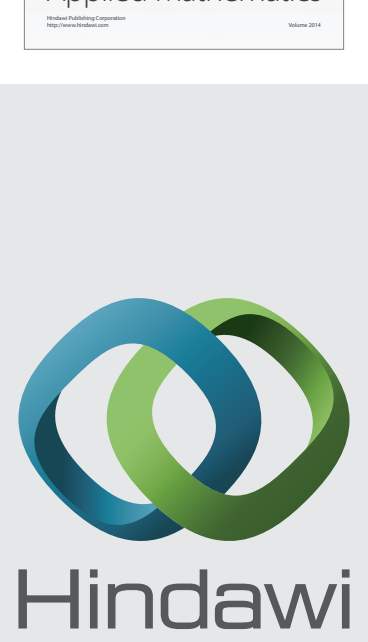

Submit your manuscripts at http://www.hindawi.com
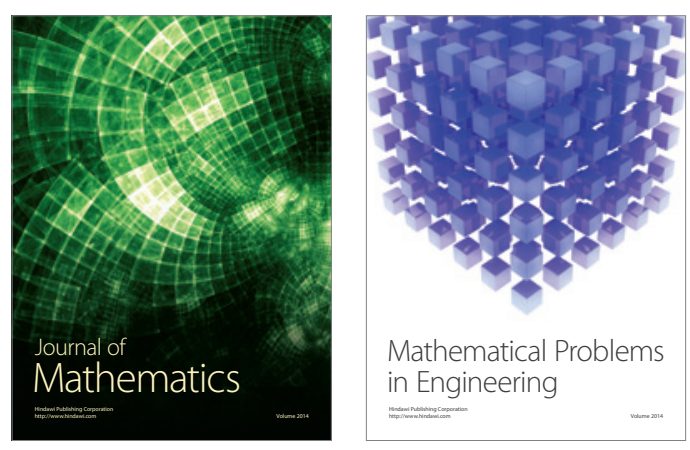

Mathematical Problems in Engineering
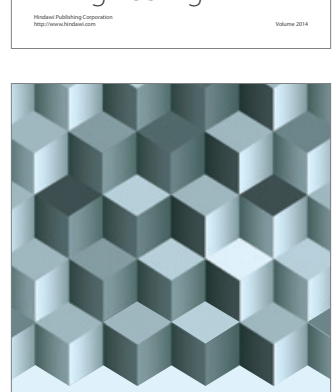

Journal of

Function Spaces
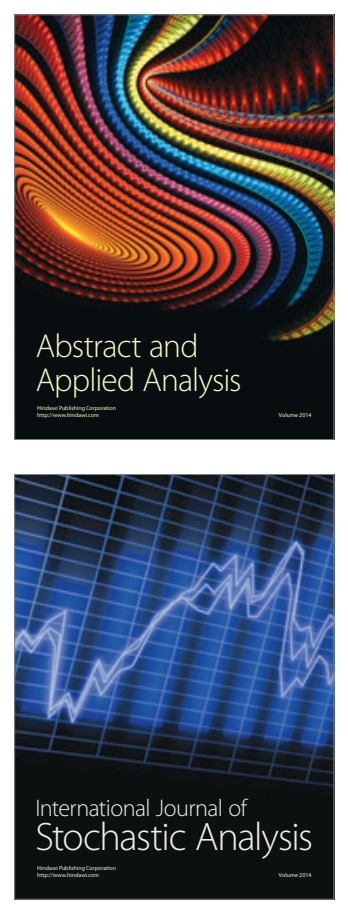

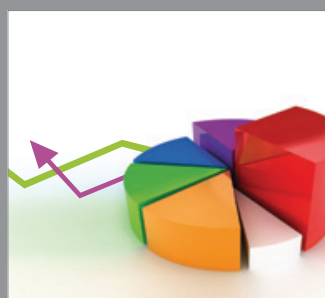

ournal of

Probability and Statistics

Promensencen
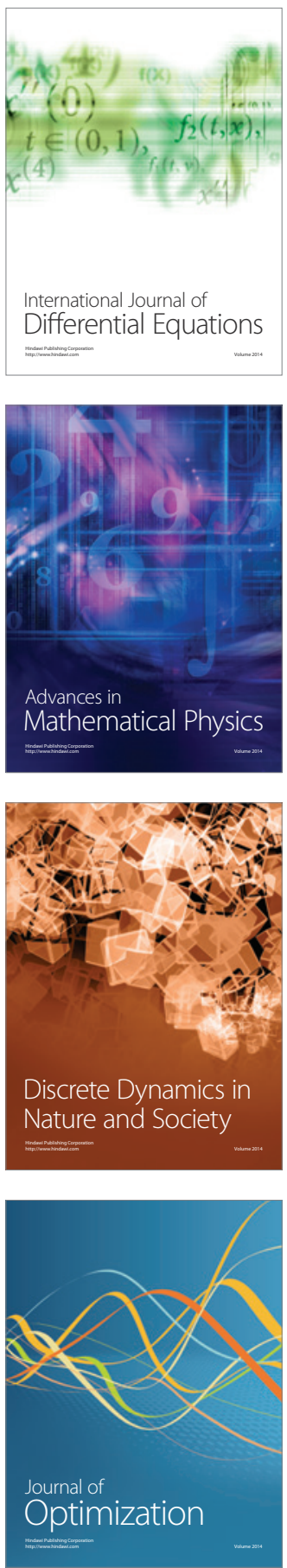